\title{
Primera cita de Alternanthera philoxerioides (Mart.) Griseb. (Amaranthaceae) en el sur de la Península Ibérica (Sevilla, España)
}

\author{
Juan Jáuregui Arana* \& Pablo García Murillo** \\ *Consejería de Agricultura, Ganadería, Pesca y Desarrollo Sostenible, Junta de Andalucía. C/. Tabladilla s/n, 41071 Sevilla \\ (España). \\ **Departamento de Biología Vegetal y Ecología, Facultad de Farmacia, Universidad de Sevilla. C/. Profesor García González \\ 2, 41012 Sevilla (España).
}

\section{Correspondencia}

Pablo García Murillo

e-mail:pgarcia@us.es

Recibido: 3 enero 2021

Aceptado: 11 febrero 2021

Publicado on-line: 18 febrero 2021

Editado por: Marta Recio Criado

\section{Resumen}

Se informa de la presencia de la especie exótica invasora Alternanthera philoxeroides en el río Guadalquivir, en Sevilla (España, S. de la Península lbérica). El hallazgo corresponde a la primera cita para Andalucía y se añade a las otras dos que ya existían para la Península lbérica.

Palabras clave: especies exóticas, corología, flora vascular, Península lbérica, plantas acuáticas, río Guadalquivir.

\section{Abstract}

Fist record of Alternanthera philoxerioides (Mart.) Griseb. (Amaranthaceae) from the Southern Iberian Peninsula (Seville, Spain)

We report the presence of the invasive alien species Alternanthera philoxeroides in the river Guadalquivir, in Seville (Spain, Southern Iberian Peninsula). This finding is the first record for Andalusia and it is added to the other two that have been already published for the Iberian Peninsula.

Key words: exotic species, chorology, vascular flora, Iberian Peninsula, aquatic plants, Guadalquivir River.
Alternanthera philoxeroides (Mart.) Griseb. Abh. Königl. Ges. Wiss.Griseb. Göttingen 24: 36. 1879; Bucholzia philoxeroides Mart., Beitr. Amarantac. 107 (1825) basion; Telanthera philoxeroides (Mart.) Moq. Prodr. 13(2): 362-363. (1849); Achyranthes philoxeroides (Mart.) Standl. J. Wash. Acad. Sci. 5 (3): 74. (1915).

ESPAÑA. Sevilla: Dársena del río Guadalquivir, meandro de San Jerónimo, 37²5'11,0"N, 5॰59'16,3"W, 7,4 msnm, 17-XII-2020, J. Jáuregui (SEV 28882).

Alternanthera philoxeroides (Amaranthaceae) (IPNI, 2020), es una hierba perenne, estolonífera, capaz de desarrollarse en medios terrestres húmedos o acuáticos (Burgin \& Norris, 2018). Arraiga en los suelos de las orillas de los cauces y extiende sus tallos huecos sobre la superficie del agua, cubriéndola y formando extensas alfombras (Sainty et al., 1997).

Su área de distribución nativa se corresponde con la cuenca del río Paraná: sur de Brasil, Bolivia, Paraguay, NE de Argentina y Uruguay (Julien et al., 1995). Sin embargo se encuentra naturalizada, como planta exótica, en prácticamente todos los continentes: en Norteamérica, se citó por primera vez en 1897 y se convirtió pronto en una plaga que originó numerosos problemas, y continúa enla actualidad en 15 estados del sur de Estados Unidos (USDA NRCS, 2020); en Asia apareció a finales del siglo XIX y en la actualidad se considera una especie invasora en varios países como: China, Birmania, Pakistán, India, Indonesia y Tailandia (Tanveer et al., 2013); en Nueva Zelanda se conoce desde 1906 y en Australia desde la década de 1940, territorios donde ha causado numerosos problemas (Julien et al., 1995, Burgin \& Norris, 2008; Basset et al., 2012). En Europa su presencia se ha observado en Francia (Fried \& Brunel, 2009) e Italia (lamonico \& Sánchez-Del Pino, 2016) y más recientemente en España, concre- tamente en Galicia (Romero \& Amigo, 2015) y Cataluña (Guardiola \& Petit, 2020).

Se trata de una especie que fuera de su área nativa, no produce semillas, o si las produce, no son viables (Julien et al., 1995); en esos lugares, se multiplica por medios vegetativos, mediante fragmentación de tallos, que pueden desgajarse fácilmente, liberando fragmentos que dispersan la planta y siendo cada nudo capaz de dar lugar a un nuevo individuo (Spencer \& Coulson, 1976). Esta capacidad de reproducirse asexualmente es considerada excepcional por Zuo et al. (2012), así como su habilidad para sobrevivir en los ambientes marginales.

Asimismo, de acuerdo con las categorías propuestas por Kornas (1990), esta planta se comporta actualmente como diáfito, es decir una especie no establecida, pero si no se toman medidas para su control, puede evolucionar rápidamente hacia epecofito, es decir naturalizarse. Ello sería consecuencia de su gran poder de propagación y crecimiento, 
rasgos que se unen a una intensa actividad alelopática, que inhibe el desarrollo de otras plantas que conviven con ella (Abbas et al., 2016; Zuo et al., 2012). Como consecuencia de todo esto, Alternanthera philoxeroides es una planta muy competitiva, con una gran facilidad para alterar, no solo los ecosistemas naturales (Bassett et al., 2012; Chatterjee \& Dewanji, 2014), sino también cultivos y áreas acuáticas recreativas (Abbas et al., 2016). Una vez establecida, resulta extremadamente difícil su erradicación, siendo este proceso, además muy costoso (Sainty et al. 1997).

Por todo ello, esta planta ha sido calificada como una de las especies exóticas invasoras más dañinas (CABI, 2020). En consecuencia, la Unión Europea, a través de su Reglamento de Ejecución 2017/1263 de la Comisión de 12 de julio de 2017, la incluyó en el listado de especies exóticas invasoras preocupantes para la Unión ("Lista de la Unión"), que contiene especies cuyo manejo y cultivo está limitado, para reducir al máximo y mitigar sus efectos adversos sobre la biodiversidad. Igualmente, a pesar de no haber estado citada en ese momentopara el territorio español, el Gobierno Español la incluyó en el Catálogo Español de Especies Exóticas Invasoras (Real Decreto 630/2013, de 2 de agosto), cuyo objetivo era prevenir la introducción de estas especies, su control y facilitar las tareas para su erradicación.

Hasta el momento se han detectado 6 subpoblaciones, todas ellas en la Dársena del Río Guadalquivir en el término de Sevilla (Fig. 1): Subpoblación 1, Meandro de San Jerónimo (37²5'11,0"N, 559'16, 3"W); Subpoblación 2, Orilla del Parque Fernando Magallanes $\left(37^{\circ} 23^{\prime} 28,6 " \mathrm{~N}, \quad 6^{\circ} 00^{\prime} 25,9 " \mathrm{~W}\right)$; Subpoblación 3, Orilla del Parque Fernando Magallanes (37²3'27,4"N, 600'25,5"W); Subpoblación 4, Orilla del Paseo de la O (37²3'23,1"N, 600'24,7"W); Subpoblación 5, Orilla del Paseo de la O $\left(37^{\circ} 23^{\prime} 20,1 " \mathrm{~N}\right.$, $\left.6^{\circ} 00^{\prime} 23,3 " W\right)$; Subpoblación 6, Orilla de Capitanía Marítima (37²2'06,5"N, 559'29,7"W).

Respecto a su procedencia, se desconoce. EPPO (2016) señala la presencia de semillas de esta especie en el alimento para aves, como posible vía de entrada y también, al agua de los depósitos de lastre de los barcos, como otra vía posible. No parece haber mucha evidencia que vincule su entrada como planta ornamental, incluida acuariofilia, en los diversos países donde no es nativa (EPPO, 2016).

Finalmente, indicar que los ecosistemas acuáticos continentales son especialmente vulnerables a las invasiones biológicas (Collen et al., 2014). El control y la erradicación de especies invasoras es más eficiente durante las primeras etapas de la invasión, así como en las primeras etapas del ciclo de vida de las plantas (Gillard et al., 2018; Rodriguez Merino et al., 2018). Por lo tanto, para evitar que esta planta colonice nuevas localidades y limitar su expansión, resulta necesaria una acción rápida por parte de la administración con competencias en la gestión de especies exóticas invasoras (Consejería de Agricultura, Ganadería, Pesca y Desarrollo Sostenible). Esta acción es imprescindible para evitar los desajustes ambientales que la invasión de esta agresiva especie exótica ha provocado en otros lugares.

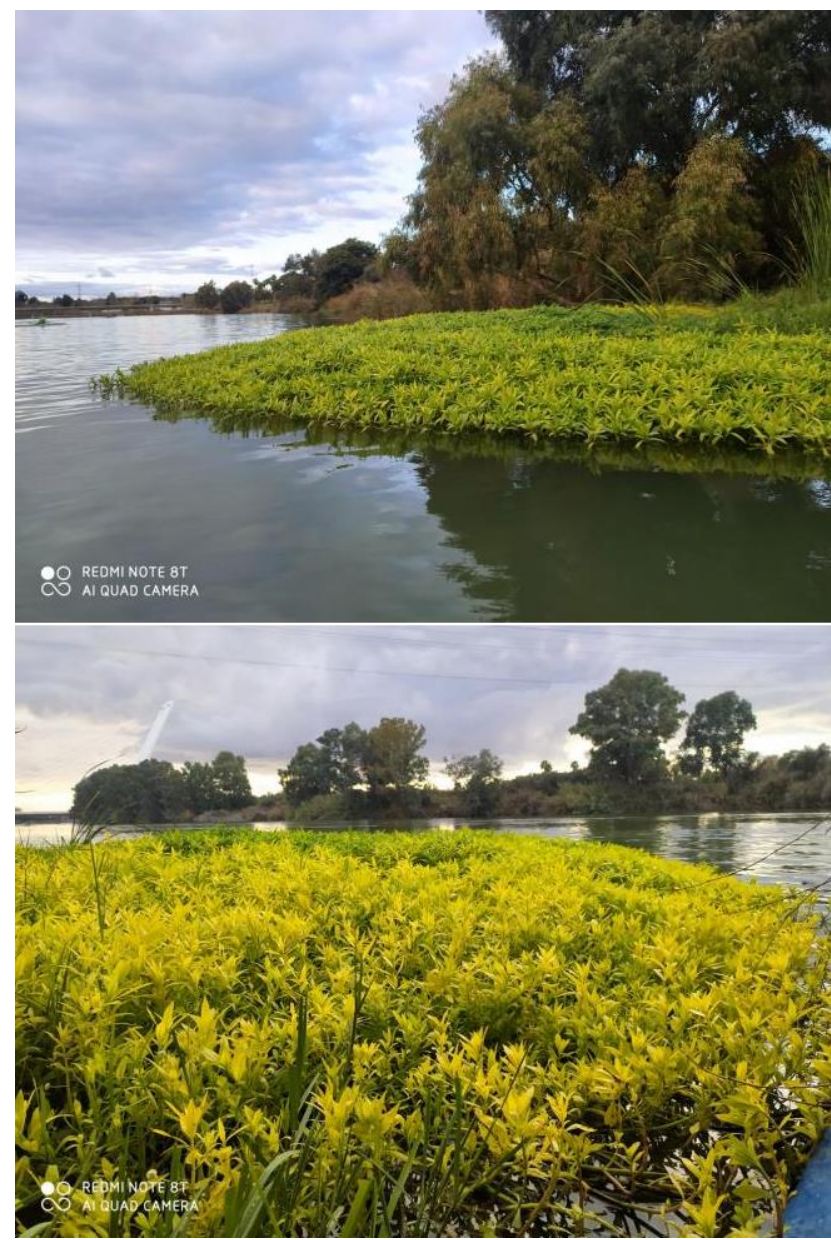

Figura 1. "Alfombras" de Alternanthera philoxeroides en la Dársena del río Guadalquivir, Sevilla (S. de la Península lbérica).

Figure 1. Alternanthera philoxeroides "carpets" in the Guadalquivir river Basin, Seville (Southern Iberian Peninsula).

\section{Bibliografía}

Abbas, T., Tanveer, A., Khaliq, A., \& Safdar, M. E. (2016). Comparative allelopathic potential of native and invasive weeds in rice ecosystem. Pakistan Journal of Weed Science Research, 22(2), 269 283.

Bassett, I., Paynter, Q., Hankin, R., \& Beggs, J. R. (2012). Characterising alligator weed (Alternanthera philoxeroides; Amaranthaceae) invasion at a northern New Zealand lake. New Zealand Journal of Ecology, 36(2), 216-222.

Burgin, S., \& Norris, A. (2008). Alligator weed (Alternanthera philoxeroides) in New South Wales, Australia: a status report. Weed Biology and Management, 8(4), 284-290.

CABI (2020). Alternanthera philoxeroides (alligator weed). In: Invasive Species Compendium. Wallingford, UK: CAB International.

www.cabi.org/isc. [consultado el 19 de diciembre de 2020].

Chatterjee, A. \& Dewanji, A. (2014). Effect of varying Alternanthera philoxeroides (alligator weed) cover on the macrophyte species diversity of pond 
ecosystems: a quadrat-based study. Aquatic Invasions, 9(3), 343-355.

Collen, B., Whitton, F., Dyer, E.E., Baillie, J.E.M., Cumberlidge, N., Darwall, W.R.T., Pollock, C., Richman, N.I., Soulsby, A.-M. \& Böhm, M. (2014). Global freshwater species congruence. Global Ecology and Biogeography, 23, 40-51.

EPPO (2016). Alternanthera philoxeroides (Mart.) Griseb. EPPO Bull, 46, 8-13.

https://doi.org/10.1111/epp.12275. [consultado el 19 de diciembre de 2020].

Fried, G. \& Brunel, S. (2009). Un nouvel outil de hierachisation des plantes exotiques: premiers résultats de son application sur une liste de 217 especès présentes en france. XIIIème Colloque International sur la Biologie des Mauvaises Herbes. Dijon, 8 - 10 Septembre 2009. Pp: 1-13.

Guardiola, M. \& Petit, A. (2020). Aportacions a la flora allòctona de la serralada Litoral central catalana i territoris propers. Butlletí de la Institució Catalana d'Història Natural, 84, 35-49.

Gillard, M., Thiébaut, G., Deleu, C. \& Leroy, B. (2017). Present and future distribution of three aquatic plants taxa across the world: decrease in native and increase in invasive ranges. Biological Invasions, 19(7), 2159-2170.

lamonico, D. \& Sánchez-Del Pino, I. (2016). Taxonomic revision of the genus Alternanthera (Amaranthaceae) in Italy. Plant Biosystems, 150(2), 333-342.

IPNI (2020). International Plant Names Index. Published on the Internet http://www.ipni.org, The Royal Botanic Gardens, Kew, Harvard University Herbaria \& Libraries and Australian National Botanic Gardens. [consultado el 18 de diciembre de 2020].

Julien, M. H., Skarratt, B. \& Maywald, G. F. (1995). Potential geographical distribution of alligator weed and its biological control by Agasicles hygrophila. Journal of Aquatic Plant Management, 33(1), 55-60.

Kornas, J. (1990). Plant invasions in Central Europe: historical and ecological aspects. In: Di Castri F., Hansen, A.J. \& Debussche, M. (eds.) Biological Invasions in Europe and the Mediterranean Basin, pp. 19-36. The Netherlands: Kluwer Academic Publishers, Dordrecht.

Rodríguez-Merino, A., García-Murillo, P., Cirujano, S. \& Fernández-Zamudio, R. (2018). Predicting the risk of aquatic plant invasions in Europe: How climatic factors and anthropogenic activity influence potential species distributions. Journal for Nature Conservation, 45, 58-71.

Romero, M. I. \& Amigo, J. (2015). Alternanthera philoxeroides (Mart.) Griseb. naturalizada en el noroeste ibérico. Nova Acta Científica Compostelana (Bioloxía), 22, 1-4.

Sainty, G., McCorkelle, G. \& Julien, M. (1997). Control and spread of Alligator Weed Alternanthera philoxeroides (Mart.) Griseb., in Australia: lessons for other regions. Wetlands Ecology and Management, 5(3), 195-201.

Spencer, N. R. \& Coulson, J. R. (1976). The biological control of alligatorweed, Alternanthera philoxeroides, in the United States of America. Aquatic Botany, 2, 177-190.

Tanveer, A., Khaliq, A. \& Siddiqui, M. H. (2013). A review on genus Alternanthera weeds implications. Pakistan Journal of Weed Science Research, 19(1), 53-58.

USDA NRCS (2020). Alternanthera philoxeroides (Mart.) Griseb. Alligatorweed. The PLANTS. National Plant Data Team, Greensboro, NC 27401-4901 USA. http://plants.usda.gov. [consultado el 19 de diciembre de 2020].

Zuo, S., Ma, Y. \& Shinobu, I. (2012). Differences in ecological and allelopathic traits among Alternanthera philoxeroides populations. Weed Biology and Management, 12(3), 123-130. 